\title{
ESTUDOS DE USUÁRIOS E PRÁTICAS INFORMACIONAIS: DO QUE ESTAMOS FALANDO?
}

\author{
ESTUDIOS DE USUARIOS Y PRÁCTICAS \\ INFORMACIONALES: ¿DE QUÉ SE TRATA?
}

\author{
Ilemar Christina Lansoni Wey Berti* \\ Carlos Alberto Ávila Araújo**
}

\begin{abstract}
RESUMO:
Introdução: O trabalho apresenta uma breve discussão acerca das diferenciações entre estudos de Comportamento informacional e Práticas informacionais presentes no campo de estudos de usuários de informação. Objetivo: Refletir sobre a relação das Práticas informacionais com o campo de Usuários da informação e propor uma análise das perguntas que as temáticas abordam e como abordam com base na Teoria social. Metodologia: Recuperam-se alguns pontos da história do desenvolvimento do campo e a influência da Teoria social para compreensão das Práticas informacionais, relacionadas à formação do conhecimento e a reciprocidade das ações na sua constituição. Resultados: Ressalta a importância da construção das significações para compreensão das necessidades, busca e uso da informação em diferentes contextos. Conclusões: As Práticas informacionais abordam as ações e significações dos sujeitos informacionais, uma perspectiva social da informação presente nos estudos de usuários.
\end{abstract}

Palavras-chave: Estudos de usuários. Práticas informacionais. Teoria social.

* Doutoranda do Programa de Pós-Graduação em Ciência da Informação da Universidade Federal de Minas Gerais (UFMG). E-mail: ilemar.berti@gmail.com.br

** Doutor em Ciências da Informação. Docente do Programa em Ciência da Informação da Universidade Federal de Minas Gerais (UFMG). E-mail: casal@eci.ufmg.br

Inf. Inf., Londrina, v. 22, n. 2, p. 389 - 401, maio/ago., 2017. 


\section{INTRODUÇÃO}

A pertinência deste ensaio justifica-se pelo constante impasse nos estudos de usuários da informação marcados pela distinção entre os estudos de Comportamento informacional e os estudos de Práticas informacionais. Localizados por diferenças no campo da teoria social e, por conseguinte, o fazer metodológico, o conceito de cultura de Geertz (1926-2006) e o conceito de "reciprocidade de perspectivas" de Schutz (1899-1959) podem contribuir para responder a evidente proposição em responder questões diversas para os estudos de usuários, concentradas nas estruturas sociais do conhecimento. Compreender como e por que as pessoas buscam informação é questão norteadora. Numa perspectiva, a investigação se concentra em como as pessoas interpretam uma determinada informação em situação específica, em outra, a importância está fundamentada em como as pessoas agem com a informação no cotidiano de suas ações, ou seja, no contexto social e cultural, dentro da visão micro e macrossociológica, de ação recíproca.

\section{ESTUDOS DE USUÁRIOS}

Para compreender as diferenças, é preciso conhecer a história do desenvolvimento do campo em questão. Os estudos de usuários estão consolidados como uma área de pesquisa em Ciência da Informação que se constituiu por dois marcos na história do uso da informação, segundo Batista e Cunha (2007). O primeiro foi na década de 1930, a partir da necessidade de se levantar características da população da cidade de Chicago, decorrente de uma imigração em massa, vinda de várias partes do mundo, e a necessidade de oferecer a estes novos moradores, por meio da biblioteca (Graduate Library School da University of Chicago) e de outros instrumentos sociais, informações com objetivo de socialização, minimizando as diferenças políticas, sociais e culturais na nova Chicago.

O segundo marco é considerado a Conferência da Royal Society de Londres de 1948, que realizou reflexões sobre a necessidade, busca e uso da 
informação por técnicos e cientistas no contexto da guerra fria, provocando importante impacto na Ciência da Informação pelo valor da própria informação, compreendida pelos fluxos informacionais, seus resultados e efeitos. O período dessa conferência foi bastante fértil para os estudos desta natureza, tratando a temática sob diferentes aspectos, uma voltada para os sistemas de informação e outra para as pessoas que utilizavam os sistemas. Estudos como esses se multiplicaram até o final da década de 1970, sem a percepção clara dos conceitos e encaminhamentos metodológicos adotados.

Segundo Ferreira (1995), as investigações do campo, com características voltadas para os usuários, inicialmente foram denominadas estudos de comunidades, estudos dos usos de informação, comportamento informacional e, posteriormente, com menor incidência, Práticas informacionais. Os termos acompanharam as diferentes formas e características de se estudar a informação sob a perspectiva dos sujeitos. No entanto, na década de 1980, estudos começaram a apontar essa distinção nas investigações. Um deles em 1986, realizado por Dervin e Nilan. Nesse estudo, os autores anunciaram que, em decorrência da evolução dos estudos de usuários, fruto da complexidade da questão informacional que envolvem os sujeitos e suas interações, duas abordagens poderiam ser identificadas, uma tradicional e uma alternativa.

A abordagem tradicional recebeu esse nome por ser inicialmente hegemônica. Como uma das formas da informação ser investigada pela Ciência da Informação, tal abordagem compreende a informação como objetiva, aplicada especialmente na avaliação de coleções, indicadores de uso e sistemas de informação, relacionada a "dado", em que os significados e importância estão em si mesmos. A abordagem tradicional dimensiona melhor a informação de forma técnica e quantitativa, a partir de tipos ou fontes de informação relacionados a taxas demográficas e perfil de usuários, desconsiderando a identificação real dos significados e impactos pessoais e sociais da informação. Essa abordagem, segundo Ferreira, não poderia ser indicadora relevante do comportamento de busca e uso da informação (FERREIRA, 1995, p.222). 
Atualmente, a abordagem alternativa tem o domínio sobre o campo por ter superado a abordagem tradicional, criticada principalmente pelo aspecto positivista, vazia de singularidade, condição peculiar dos seres humanos. Portanto, em resposta às necessidades de estudos que posicionassem os usuários no centro do processo de busca da informação, surgiu a abordagem alternativa, como crítica ao modelo tradicional, ao considerar os usuários apenas como processadores da informação. $\mathrm{Na}$ abordagem alternativa, a partir da década de 1970, várias teorias e modelos explicativos foram usados para discutir a questão da necessidade de informação, representados por investigações ligadas especialmente ao Comportamento informacional dos usuários.

No primeiro momento, a abordagem alternativa não contemplou a perspectiva das Práticas Informacionais, esse conceito apareceu posteriormente, discutido por autores como Savolaine (1995), Tajla (1996) e Wilson (2002) a partir do início da década de 1990, sem muita incidência no campo. Estes autores, ancorados numa "epistemologia social" da informação, defendida por Hjorland (2002), Capurro (2003), Rendón Rojas (2005) e Frohmann (2008), postulam que o objeto "informação" contempla além dos aspectos físicos e cognitivos, o aspecto social. A afirmação corresponde às construções sociais que levam em conta o caráter individual, coletivo, cultural, político e ideológico, de uma realidade construída reciprocamente, distinta teórica e metodologicamente do proposto nos paradigmas físicos e cognitivos, conforme Capurro (2003). Portanto, o entendimento a partir da epistemologia social afeta o campo de usuários, que embasa o conceito de Práticas informacionais, conduzindo as investigações para além da ideia de Comportamento informacional em que os sujeitos agem a partir de estímulos externos, calcado no behaviorismo do ponto de vista cognitivo.

\section{COMPORTAMENTO INFORMACIONAL}

O conceito de Comportamento Informacional, portanto, está relacionado com a identificação da necessidade de informação, percebida pelos sujeitos 
que estão em busca de resolver seus problemas informacionais. Conforme Wilson (2000), um dos principais pesquisadores do Comportamento de usuários na busca por informação, a pessoa que sente necessidade de uma informação e a identifica pode tomar diversos caminhos, que são influenciados por fatores internos e externos, discutidos nos diferentes modelos desenvolvidos na área, entre eles, os de Dervin (1983), de Taylor (1986) e de Kuhlthau (1991).

A abordagem de Dervin (1983), chamada de Sense-making, atribuiu a condição da necessidade de informação à incompletude no sentido de falta, de lacuna de uma determinada situação. A partir de uma realidade empírica, a teoria do Sense-making pode ser compreendida metaforicamente como uma "peça" do pensamento e sentimento do usuário diante da falta (lacuna) que uma pessoa pode ter na sua realidade. Amplamente utilizada como modelo de busca da informação, esta teoria aborda a criação de significado da necessidade informacional. Dervin (1983) utilizou uma metáfora baseada em quatro itens constituintes: situação, lacuna, uso e estratégias utilizadas para transpor a situação. A teoria do Sense-making aborda a criação de significado representada pelo vazio cognitivo e o uso da informação para preenchê-lo por meio de estratégias utilizadas pelo indivíduo.

A abordagem de Taylor discute a informação útil ao usuário que a busca a partir da atribuição de valor instalada nos processos de seleção, análise e julgamento, aplicados nas tomadas de decisão e ações pessoais. Taylor (1986), em seu modelo, acrescentou a dimensão ambiental ao processo de busca e uso da informação. Nesse contexto, a interação com as pessoas é fundamental, de acordo com o autor, esse pode ser fator determinante de condições e características do processo de busca e uso da informação. São quatro as categorias inclusas no processo: o grupo, as características comuns dos problemas encontrados, o convívio e os condicionantes ambientais.

A abordagem de Kuhlthau (1991) propõe um modelo de observação de busca da informação por etapas: início, seleção, exploração e formulação, além dos aspectos cognitivos e emocionais, como sentimento de incerteza, otimismo e satisfação. Esse modelo considera elementos emocionais ao processo de 
busca da informação, compreendendo que as necessidades informacionais estão imbuídas de sentimentos, além do pensamento racional. Segundo Kuhlthau (1991), essa teoria aborda estágios atribuídos à busca informacional, nos quais o sentimento de incerteza é destacado dentro do processo e pode variar ao longo das etapas. A proposta de acrescentar a dimensão emocional considera que esses sentimentos influenciam no processo de busca e na forma com que as pessoas usam a informação.

Muitos outros modelos poderiam ser descritos para evidenciar os pontos divergentes entre Comportamento informacional e Práticas informacionais. Criados com o objetivo de explicar como os usuários buscam a informação a partir de uma necessidade consciente, os modelos relacionam o comportamento a uma situação que o usuário se encontra especialmente no contexto da pesquisa, em bibliotecas ou espaços especializados. Estes modelos tornaram-se importantes referências para responder questões ligadas aos caminhos tomados pelas pessoas que necessitam de informação. Contudo, as pessoas, usuárias de informação em seu cotidiano, não operam apenas quando têm uma necessidade, mas em toda ação informacional, relacionada ao conceito de informação pragmático, humanístico, baseado em relacionamentos, interações, linguagens convencionadas, marcadas pelo social construído reciprocamente.

\section{PRÁTICAS INFORMACIONAIS}

Conforme descrito anteriormente, no desenvolvimento das investigações do campo, alguns pesquisadores começaram a verificar que os modelos de comportamento não correspondiam a muitas situações do cotidiano, como as investigadas por Talja (1996) e McKenzie (2003), em que a necessidade não era explícita e a busca pela informação estava relacionada ao contexto social e cultural. A partir nessa perspectiva, as Práticas informacionais precisam ser compreendidas do ponto de vista de outras áreas do conhecimento, como a antropologia e a sociologia que atribuem para a compreensão da realidade social a concepção da construção contínua, amplamente influenciada, sendo 
necessárias considerações mais complexas em virtude da impossibilidade de previsão e determinação de uma única resposta.

As Práticas informacionais representam a busca por informação pautada na relação informacional influenciada pelas interações sociais, de modo que compreendem os usuários e a informação em espaços diferentes, independentes, porém recíprocos. Os valores dados a informação pelos sujeitos podem ser entendidos pela cultura semiótica, de que ao mesmo tempo que o usuário recorre aos significados culturais, contribui para produzi-los e reforçá-los como consideram Geertz (1978) e Wagner (1975). Em concordância à condição da interação do usuário com a informação, com base nas construções históricas e sociais dos sujeitos, está o paradigma social da informação de Capurro (2003), o enfoque pragmático de Rendón Rojas (2005) e a abordagem interacionista defendida por Araújo (2012).

$\mathrm{Na}$ teoria social a constituição das Práticas informacionais é igualmente reforçada pela reciprocidade das significações ao destacar a interação como 'ação recíproca'. O conceito põe em relevo o fato de uma ação ou influência exercida por algo ser também afetada por esse algo. Numa perspectiva interacionista, pragmática, o usuário não é totalmente determinado pelo contexto em que está inserido, nem é totalmente isolado ou alheio a ele; a determinação que o contexto exerce existe, é real, mas não é mecânica nem absoluta, é interpretada e alterada pelo sujeito (ARAÚJO, 2012, p.149). Para Araújo, a interação está presente no movimento de busca, seleção e atribuição de valor à informação, tem origem no seu ambiente social e sofre interferência dos contextos socialmente construídos, decorrentes das marcações coletivas descritas por Roy Wagner como uma invenção social (WAGNER, 1975, p.108).

Contudo, os contextos sociais também são influenciados a partir das relações estabelecidas por esse mesmo usuário, alterando os processos de busca e uso da informação. Nas investigações sob essa vertente, a interação caracteriza a complexidade percebida nos estudos de usuários e suas dimensões individuais, coletivas, sociais, culturais e políticas, possíveis de serem apreendidas no espaço social. A compreensão, portanto, frente aos estudos de usuários da Ciência da Informação, é que a informação não é 
somente determinada por um fator externo que se ajusta perfeitamente às necessidades, de acordo com o estudo do Comportamento informacional propõe, há um conjunto de fatores humanos, pessoais, individuais, coletivos que determinam sua aderência, de maneira que suas características são microssociológicas, melhor dizendo, propondo-se ao exercício de olhar para o micro para responder ao macrossocial. .

A proposta metodológica da teoria social propõe um olhar fenomenológico para as Práticas informacionais, possível de encontrar em Alfred Schutz (1979). A perspectiva do autor é a observação pelo enfoque do conhecimento constituído na intersubjetividade, ou seja, na partilha das significações, uma prática essencialmente hermenêutica e dialética que busca respostas nas convenções da realidade social que Schutz (1979) designa como a "tese geral da reciprocidade de perspectivas". Para o autor a reciprocidade realiza-se por meio de duas idealizações, são elas, a troca de pontos de vista, ligada a apreensão da tipificação e a idealização da congruência ou conformidade dos sistemas de pertinência, de modo a ligar intrinsecamente o indivíduo da ação ao outro da situação vigente.

A necessidade de considerar a informação ligada ao mundo que as pessoas vivem e dão sentido as suas ações, ou seja, as questões devem ser dirigidas ao contexto e não somente ao modo de pensar das pessoas. Embora - aspecto racional do paradigma cognitivo responda a questões do Comportamento informacional, não é suficiente para explicar as condutas e significações humanas atribuídas aos fenômenos informacionais. As dimensões dessa abordagem estudada por autores como Capurro (2003), descrita como uma "epistemologia social", abarca a teoria social de sustentação das práticas de pesquisa ligadas ao modo como se opera o olhar ao objeto informacional. Por ser um sistema de signos convencionados interpretáveis, a sociologia e mesmo a cultura não é um poder, algo a que podem ser atribuídos casualmente os acontecimentos sociais, os comportamentos, as instituições ou os processos, trata, portanto, de atribuições situacionais.

Compreender como que os atores sociais produzem seus significados, 
conduz à análise sociológica como forma de conhecimento, sem, no entanto, limitá-la a um método. O lugar ocupado pelas construções da realidade social, representa um campo simbólico rico para a investigação das ações informacionais, sendo este propício para a percepção da formação do conhecimento. Nesse contexto, a informação é entendida a partir das relações de construção interpretativa, conforme as experiências pessoais de cada indivíduo, e da representação de significação e dimensão intersubjetiva, baseada nas interações múltiplas, próprias dos seres humanos. À medida que as informações provocam a ação mental do indivíduo e passam a ser aplicadas em situações do cotidiano, reforçam $o$ arsenal social e cultural institucionalizado como considera Marteleto (1995), por meio do funcionamento sócio-histórico.

\section{RESULTADOS}

A temática Prática informacionais, relaciona a necessidade de informação dos usuários e envolve as práticas de busca e uso da informação nos mais diversos contextos. No entanto, partimos do pressuposto de que a informação não é um objeto adaptável, em virtude de cada ser humano ser único em suas necessidades, o que provoca uma ampla complexidade discutida independente dos ambientes formais e institucionalizados, capaz de considerar os aspectos dinâmicos da informação e dos fundamentos sociais.

Essa abordagem dá ênfase ao terceiro paradigma reconhecido por Capurro como "paradigma social", o qual ele considera o que melhor representa a informação como conhecimento em ação, ligado à maneira como as pessoas compartilham sua realidade umas com as outras. Frohmann (2008) também critica as abordagens que tratam o conceito da informação unicamente mentalista, reforçando a ideia da materialidade da informação para o caráter social da informação. Os estudos na perspectiva da análise do domínio de Hjørland e Albrechtsen (1995) relacionam a especificidade dos sujeitos e a intersubjetividade que denotam o caráter construtivo da informação, no qual se dá a dimensão pragmática dos fluxos informacionais, possibilitando observar o 
objeto também por abordagens fenomenológicas, interpretando a informação não como coisa, mas como processo, construída histórica e culturalmente, podendo ser vista dessa forma apenas pela perspectiva dos sujeitos.

A informação é multidimensional e a investigação das propriedades e das Práticas informacionais, seu fluxo, processamento e aplicação é o grande desafio para os estudos da área. Morin (1984, p.93), em concordância com este apontamento e reforçando a complexidade da questão, considera o conceito de informação um "encruzilhado", sendo necessária a aproximação de outros conceitos e fenômenos para dar respostas à análise da realidade social.

Portanto, teorias que embasam modelos, em detrimento da compreensão de como se constroem os significados, valorizam os processos informacionais numa visão unidimensional do pensar, principal crítica aos estudos de Comportamento informacional, pois promovem um afastamento das representações simbólicas observadas no campo da interação social. Nota-se, portanto, como pressuposto da perspectiva das Práticas Informacionais, a ação do indivíduo como aponta Talja (1996), abarcando as explicações da natureza do conhecimento a partir dos fenômenos sociais, valorizando as estruturas de formação do conhecimento e não do homem - informação apontada pela teoria cognitiva.

\section{REFERÊNCIAS}

ARAÚJO, Carlos Alberto Ávila. Paradigma Social nos estudos de usuários da informação: abordagem interacionista. Informação \& Sociedade: Estudos, João Pessoa, v.22, n.1, p. 145-159, jan./abr., 2012.

BAPTISTA, Sofia Galvão; CUNHA, Murilo Bastos da. Estudo de usuários: visão global dos métodos de coleta de dados. Perspectivas em Ciência da Informação, Belo Horizonte, v. 12, n. 2, 2007. Disponível em:<http://www.scielo.br/scielo.php? script=sci arttext\&pid=S141399362007000200011\&lng=en\&nrm=iso>. Acesso em: 07 jul. 2016. 
CAPURRO, Rafael. Epistemologia e Ciência da Informação. In: ENCONTRO NACIONAL DE PESQUISA EM CIÊNCIA DA INFORMAÇÃO, 5., 2003, Belo Horizonte. Anais...Belo Horizonte: Associação Nacional de pesquisa e Pós graduação em Ciência da Informação e Biblioteconomia, 2003.

DERVIN, Brenda; NILAN, Michael. Information needs and uses. Annual review of information science and technology. White Plains, NY: Knowledge Industry Publications, 1986.

FERREIRA, Sueli Mara Soares Pinto. Novos paradigmas e novos usuários de informação. Ciência da Informação, Brasília, v. 25, n. 2, p. 1-10, 1995.

FROHMANN, Bernd. O caráter social, material e público da informação. In: FUJITA, Mariangela Spotti Lopes; MARTELETO, Regina Maria; LARA, Marilda Lopes Ginez (Org.). A dimensão epistemológica da ciência da informação e suas interfaces técnicas, políticas e institucionais nos processos de produção, acesso e disseminação da informação. São Paulo: Cultura Acadêmica; Marília: Fundepe, 2008.

GEERTZ, Clifford. A interpretação das culturas. Rio de Janeiro: Zahar, 1978.

HJORLAND, Biger; Albrechtsen, Hanne. Toward a new horizon in information science: domain analysis. Journal of the American Society for Information Science, Washington, v. 46, n.6, p. 400-425, 1995.

HJORLAND, Biger. Epistemology and the socio-cognitive perspectives in Information Science. Journal of the American Society for Information Science and Technology, New York, v.53, n.4, p. 257-270, 2002.

KUHLTHAU, Carol. Inside the search process: information seeking from the users perspective. Journal of the American Society for Information Science, Washington, v.42, n. 5, p. 361-371, 1991.

MCKENZIE, Pamela. A model of information practices in accounts of everyday - life information seeking. Jornal of Documentation, v. 59, n. 1, p. $19-40$, 2003.

MORIN, Edgar. Sociologie. Paris: Fayard, 1984.

MARTELETO, Regina. Cultura informacional: construindo o objeto informação pelo emprego dos conceitos de imaginário, instituição e campo social. Ciência da Informação, Brasília, v. 24, n.1, 1995.

RENDÓN ROJAS, Miguel. Relación entre los conceptos: información, conocimiento y valor. Semejanzas y diferencias. Ciência da Informação, Brasília, v. 34, n. 2, p. 52-61, maio/ago. 2005.

SCHÜTZ, Alfred. Fenomenologia e relações sociais. Rio de Janeiro: Zahar Editores, 1979. 
TAYLOR, Robert S. Professional aspects of information science and technology. In: ANNUAL REVIEW OF INFORMATION SCIENCE AND TECHNOLOGY-ARIST, v.1, p. 15-40, 1986.

TALJA, Sanna. Constituting "Information" and "User" as Research Objects: A Theory of Knowledge Formations as an Alternative to the Information Man Theory. In: VAKKARI, Perti; SAVOLAINEN, Reijo; DERVIN, Brenda (Eds). Information Seeking in Context. Londres: Taylor Graham, 1996, p.67-80.

WAGNER, Roy. A invenção da cultura. São Paulo: Cosac Naify Portátil, 1975.

WILSON, Thomas Daniel. Human Information Behavior. Informing Science, Brookhill Court, v. 3, n. 2, p. 49-54, 2000.

. On User Studies and Information Needs. Journal of Documentation, London, v. 62, n. 6, p. 658-670, 2006. Disponívem em: <http://wwwemeraldinsightcom.ez27. periodicos. capes.gov.br/journals. $h$ tm? issn $=00220418 \&$ volume $=62 \&$ is sue $=6 \&$ articleid $=1580623 \&$ show $=$ pdf $>$. Acesso em: 6 de maio 2013 .

WILSON, Tom. A dimensão epistemológica da Ciência da Informação e seu impacto sobre o ensino em Arquivologia e Biblioteconomia. Brazilian Journal of Information Science, Marília, v. 2, n. 1, p. 3-15, jan./jun. 2008. Disponível em: <http://www2.marilia.unesp.br/revistas/index.php/bjis/article/view/43/2046>. Acesso em: 6 maio 2013.

Title

User Studies and Information Practices: What we are talking about?

\begin{abstract}
Introduction: This paper presents a brief discussion on differences between Information behavior and Information practices, concerning the field of Studies of information users. Objective: Reflect on the relationship of Information Practices with the field of Studies of information users and propose the analysis of questions that these topics cover, and how it is addressed with basis on Social theory. Methodology: Some points of history of field evolution and the influence of Social theory to comprehension of Information Practices are recovered, related to the knowledge generation and reciprocity of actions in its constitution. Results: It gives light to the importance of constructions of meanings to the comprehension of needs, search and use of information in distinct contexts. Conclusions: Information practices address the actions and meanings of informational subjects, a social perspective of information present in user studies.
\end{abstract}

Keywords: User studies. Informational practices. Social theory. 


\section{Titulo}

Estudios de Usuarios y Prácticas Informacionales: ¿De qué se trata?

\section{Resumen}

Introducción: El trabajo presenta una discusión breve acerca de las diferencias entre los estudios del Comportamiento informacional y las Prácticas informacionales presentes en el campo de estudios de los usuarios de la información. Objetivo: Reflexionar sobre la relación de las Prácticas informacionales y el campo de Usuarios de la información y proponer un análisis de las preguntas que las temáticas abordan con base en la Teoría social. Metodología: Se recuperan algunos puntos de la historia del desarrollo del campo y de la influencia de la Teoría social para comprender las Prácticas informacionales relacionadas a la formación del conocimiento y a la reciprocidad de las acciones en su constitución. Resultados: Hace hincapié en la importancia de la construcción de las significaciones para la comprensión de las necesidades, en la búsqueda y en el uso de la información en diferentes contextos. Conclusiones: Las Prácticas informacionales tratan sobre las acciones y significaciones de los sujetos informacionales, una perspectiva social de la información presente en los estudios de usuarios.

Palabras clave: Estudios de Usuarios. Prácticas Informacionales. Teoría Social.

Recebido: 30.08 .2017

Aceito: 30.09 .2017

Inf. Inf., Londrina, v. 22, n. 2, p. 389 - 401, maio/ago., 2017. 